\title{
Distribution of Time-Averaged Observables for Weak Ergodicity Breaking
}

\author{
A. Rebenshtok, E. Barkai \\ Department of Physics, Bar Ilan University, Ramat-Gan 52900 Israel
}

\begin{abstract}
We find a general formula for the distribution of time-averaged observables for systems modeled according to the sub-diffusive continuous time random walk. For Gaussian random walks coupled to a thermal bath we recover ergodicity and Boltzmann's statistics, while for the anomalous subdiffusive case a weakly non-ergodic statistical mechanical framework is constructed, which is based on Lévy's generalized central limit theorem. As an example we calculate the distribution of $\bar{X}$ : the time average of the position of the particle, for unbiased and uniformly biased particles, and show that $\bar{X}$ exhibits large fluctuations compared with the ensemble average $\langle X\rangle$.
\end{abstract}

PACS numbers: 05.70.Ln, 05.20.Gg, 05.40.Fb

A central pillar of statistical mechanics is the ergodic hypothesis; which yields the equivalence of time and ensemble averages in the limit of long measurement time $t$. The Deborah number $D_{\mathrm{e}}=t_{p} / t$ is the ratio of the time scale of relaxation of the physical phenomenon under observation $t_{p}$ and the time of observation [1]. For a system to exhibit ergodic behavior $D_{\mathrm{e}}$ must be small. Recently there is much interest in weak ergodicity breaking [2], where the Deborah number diverges [3, 4, 5, 6, 7]. Weakly non-ergodic behavior is found in systems whose dynamics are characterized by power law distributed sojourn times in micro-states of the system, in such a way that the averaged waiting time is infinite (i.e. scale free dynamics). Weak ergodicity breaking was investigated for blinking quantum dots [3], intermittent nonlinear maps generating sub-diffusion deterministically [5], numerical simulations of fractional transport in a washboard potential [6] and in vivo gene regulation by DNAbinding proteins [7]. On the stochastic level, all these systems are modeled using the well known continuous time random walk (CTRW) approach or the corresponding fractional Fokker-Planck equation [8, 9, 10, 11]. Previously, non-trivial statistics of occupation times for the CTRW model were found, and it is well established that time averages remain random variables even in the limit of long measurement time [4]. The main open theoretical challenge is to find the distribution of time averages of physical observables. Such a general theory, presented in this manuscript, gives analytical estimates for the statistical deviations of time averages from ensemble averages. The theory replaces standard ergodic statistical mechanics, and is applicable for a wide class of systems modeled using the CTRW or the related fractional Fokker-Planck equation.

We consider the one-dimensional CTRW on a lattice, with lattice points $x=1, \cdots, L$. After waiting the particle can jump to one of its nearest neighbors, with probability $q_{x}$ it jumps to its left, and with probability $1-q_{x}$ to its right. The waiting times on lattice cells are independent identically distributed random variables with a common probability density function (PDF) $\psi(\tau)$. We consider the widely applicable case
8. 9, 10, 11, 12], where the PDF of the waiting times behaves like $\psi(\tau) \sim A_{\alpha} \tau^{-1-\alpha} /|\Gamma(-\alpha)|$ with $0<\alpha<1$, $A_{\alpha}>0$ when $\tau \rightarrow \infty$. In this case the average waiting time is infinite and the Deborah number diverges. Such waiting times yield anomalous sub-diffusion and are well investigated $[8,9,10,11,12$, in the context of chaotic dynamics 13], geophysics 14], sub-diffusive chemical reactions which are important in biological applications 15], and charge transport in amorphous semi-conductors 16], to name a few examples. The vast literature on the CTRW, deals mainly with ensemble averages of physical observables, for example the behavior of the ensemble average of the coordinate $\langle X\rangle$ was thoroughly investigated in many physical situations. Here we investigate the time averages, for example we will find the distribution of $\bar{X}$.

Two types of CTRWs are considered. Thermal random walks describe a physical situation where the particle is coupled to a thermal heat bath with a temperature $T$ 9, 10]. In this case the jump probabilities $q_{x}$ satisfy usual detailed balance conditions which relate $q_{x}$ with an external force field $F(x)$ acting on the system, and temperature $T$ 4, 9, 10]. When these conditions are imposed on the dynamics an ensemble of non-interacting particles attains Boltzmann equilibrium. A second class of random walks is non-thermal and this situation may describe a system far from thermal equilibrium. In this case the ensemble reaches an equilibrium which depends of-course on the transition probabilities $q_{x}$ (see details below). We will treat the non-ergodicity for both cases.

We introduce two types of measurements which we identify with two different types of ensembles. In the first the time average of a physical observable is made for a fixed time $t$ and $t \rightarrow \infty$. Repeating the experiment many times, on an ensemble of trajectories, the distribution of the time average is constructed. In the second approach the number of jumps $n$ the particle makes is fixed and $n \rightarrow \infty$. So in the first ensemble, time is fixed and $n$ fluctuates, while the opposite situation describes the second case. The fixed $n$ fluctuating time ensemble is very convenient for calculations, and as we discuss below yields the same results as the fixed time fluctuating $n$ approach. 
We begin the analysis by considering the random walk where $n$ is the operational time. The probability of occupying lattice site $x$ after $n$ jumps is given by the discrete time master equation

$$
P_{x}(n+1)=q_{x+1} P_{x+1}(n)+\left(1-q_{x-1}\right) P_{x-1}(n) \text {. }
$$

After many jumps $n \rightarrow \infty$ an equilibrium $P_{x}^{\mathrm{eq}}(n+1)=$ $P_{x}^{\text {eq }}(n)$ is obtained, which satisfies

$$
P_{x}^{\mathrm{eq}}=q_{x+1} P_{x+1}^{\mathrm{eq}}+\left(1-q_{x-1}\right) P_{x-1}^{\mathrm{eq}} .
$$

Such an equilibrium does not depend on the initial condition of the system [17], and is reached provided that the system is finite, and that $q_{x} \neq 1 q_{x} \neq 0$ besides on the boundaries.

We consider the number ensemble where $n$ is fixed. The time $t_{x}$ spent by the particle in lattice cell $x$ is called the occupation time. The total measurement time is $t=$ $\sum_{x=1}^{L} t_{x}$. According to the CTRW model the time $t_{x}$ is a sum of independent identically distributed sojourn times with the common power law tailed PDF $\psi(\tau)$. Let $n_{x}$ be the number of sojourn times in cell $x$, which is clearly large when $n \rightarrow \infty$. For the discrete time random walk described by Eq. (1) we have $n_{x} / n=P_{x}^{\text {eq }}$. Hence we may use Lévy's generalized central limit theorem and obtain the PDF of $t_{x}$

$$
f\left(t_{x}\right)=l_{\alpha, A_{\alpha} P_{x}^{\mathrm{eq}} n}\left(t_{x}\right),
$$

where the one sided Lévy PDF Eq. (3) is in Laplace $t_{x} \rightarrow$ $u_{x}$ space $\hat{f}\left(u_{x}\right)=\exp \left(-A_{\alpha} P_{x}^{\text {eq }} n u_{x}^{\alpha}\right)$. For the ergodic case, $\alpha=1$ in Eq. (3), we have $f\left(t_{x}\right)=\delta\left(t_{x}-P_{x}^{\text {eq }}\langle\tau\rangle n\right)$, where $\langle\tau\rangle=A_{1}$ is the averaged waiting time, and since $n\langle\tau\rangle \rightarrow t$ we have $f\left(t_{x}\right)=\delta\left(t_{x}-P_{x}^{\mathrm{eq}} t\right)$, as expected.

The time average of a physical observable $\overline{\mathcal{O}}$ is

$$
\overline{\mathcal{O}}=\sum_{x=1, L} \bar{p}_{x} \mathcal{O}_{x}
$$

where $\bar{p}_{x}=t_{x} / t$ is the occupation fraction and $\mathcal{O}_{x}$ is the value of the physical observable when the particle is in state $x$. For example if the observable $\mathcal{O}$ is the position $X$ of the particle we have $\bar{X}=\sum_{x=0}^{L} x \bar{p}_{x}$. For usual ergodic systems and in the long time limit $\bar{p}_{x}=P_{x}^{\text {eq }}$ and then the time average is equal to the ensemble average $\overline{\mathcal{O}}=\langle\mathcal{O}\rangle=\sum_{x=1, L} P_{x}^{\mathrm{eq}} \mathcal{O}_{x}$. When $\alpha<1$ the dynamics is non-ergodic and $\overline{\mathcal{O}}$ is a random variable, even in the long time limit.
To obtain the distribution of $\overline{\mathcal{O}}$ we find now the $L$ dimensional joint PDF of the occupation fractions $P_{L}\left(\bar{p}_{1}, \cdots \bar{p}_{x}, \cdots \bar{p}_{L}\right)[18]$. First note that the $L$ occupation fractions $\bar{p}_{x}$ are constrained according to the condition $\sum_{x=1}^{L} \bar{p}_{x}=1$, hence

$P_{L}\left(\bar{p}_{1}, \cdots, \bar{p}_{L}\right)=\delta\left(1-\sum_{x=1}^{L} \bar{p}_{x}\right) \int_{0}^{\infty} g\left(\bar{p}_{1}, \cdots \bar{p}_{L-1}, t\right) \mathrm{d} t$,

where $g\left(\bar{p}_{1}, \cdots, \bar{p}_{L-1}, t\right)$ is the $L$ dimensional joint PDF of the random variables in its parenthesis. Since the occupation times $t_{x}$ are all independent we have

$$
g\left(\bar{p}_{1}, \cdots, \bar{p}_{L-1}, t\right)=
$$

$\frac{\partial\left(t_{1}, \cdots, t_{L-1}, t\right)}{\partial\left(\bar{p}_{1}, \cdots, \bar{p}_{L-1}, t\right)}\left[\Pi_{x=1}^{L-1} l_{\alpha, A_{\alpha} P_{x}^{\mathrm{eq}} n}\left(t_{x}\right)\right] l_{\alpha, A_{\alpha} P_{L}^{\mathrm{eq}} n}\left(t-\sum_{x=1}^{L-1} t_{x}\right)$

Calculating the Jacobian we obtain $g\left(\bar{p}_{1}, \cdots, \bar{p}_{L-1}, t\right)$, then using Eq. (5) and the identity

$$
l_{\alpha, A_{\alpha} P_{x}^{\mathrm{eq}} n}\left(t_{x}\right)=\frac{1}{\left(A_{\alpha} n\right)^{1 / \alpha}} l_{\alpha, P_{x}^{\mathrm{eq}}}\left(\frac{t_{x}}{\left(A_{\alpha} n\right)^{1 / \alpha}}\right)
$$

we find

$P_{L}\left(\bar{p}_{1}, \cdots, \bar{p}_{L}\right)=\delta\left(1-\sum_{x=1}^{L} \bar{p}_{x}\right) \int_{0}^{\infty} \mathrm{d} y y^{L-1} \Pi_{x=1}^{L} l_{\alpha, P_{x}^{\mathrm{eq}}}\left(y \bar{p}_{x}\right)$.

This Eq. is the key for the calculation of the distribution of the time average $\overline{\mathcal{O}}$, as we will soon show. The multidimensional PDF of the occupation fractions Eq. (8) is independent of the number of steps $n$, and the detailed shape of the waiting time besides $\alpha$ of-course (e.g. $A_{\alpha}$ is not important). A derivation of Eq. (8) using the fixed time ensemble will be presented in a longer publication.

To proceed we investigate the characteristic function $\left\langle e^{-u \sum_{x=1}^{L} \mathcal{O}_{x} t_{x}}\right\rangle_{t}$ of the random variable $\sum_{x=1}^{L} \mathcal{O}_{x} t_{x}$ in Laplace $t \rightarrow s$ space

$$
\left\langle e^{-u \sum_{x=1}^{L} \mathcal{O}_{x} t_{x}}\right\rangle_{s}=\int_{0}^{\infty} e^{-s t}\left\langle e^{-u \sum_{x=1}^{L} \mathcal{O}_{x} t_{x}}\right\rangle_{t} \mathrm{~d} t .
$$

Using Eq. (8) we obtain

$$
\left\langle e^{-u \sum_{x=1}^{L} \mathcal{O}_{x} t_{x}}\right\rangle_{s}=\int_{0}^{\infty} \mathrm{d} t \int_{0}^{\infty} \mathrm{d} y \int_{0}^{\infty} \mathrm{d} t_{1} \cdots \int_{0}^{\infty} \mathrm{d} t_{L} t \delta\left(t-\sum_{x=1}^{L} t_{x}\right) y^{L-1} e^{-s t-u \sum_{x=1}^{L} \mathcal{O}_{x} t_{x}} \Pi_{x=1}^{L} l_{\alpha, P_{x}^{\mathrm{eq}}}\left(y t_{x}\right)=
$$




$$
-\frac{\mathrm{d}}{\mathrm{d} s} \int_{0}^{\infty} \mathrm{d} y y^{L-1} \int_{0}^{\infty} \mathrm{d} t_{1} \cdots \int_{0}^{\infty} \mathrm{d} t_{L} e^{-s \sum_{x=1}^{L} t_{x}-u \sum_{x=1}^{L} \mathcal{O}_{x} t_{x}} \Pi_{x=1}^{L} l_{\alpha, P_{x}^{\text {eq }}}\left(y t_{x}\right)=
$$

$$
-\frac{\mathrm{d}}{\mathrm{d} s} \int_{0}^{\infty} \mathrm{d} y y^{L-1} \Pi_{x=1}^{L}\left\{\frac{\exp \left[-P_{x}^{\mathrm{eq}}\left(\frac{s+\mathcal{O}_{x} u}{y}\right)^{\alpha}\right]}{y}\right\} .
$$

Solving the last integral we find the characteristic function

$$
\left\langle e^{-u \sum_{x=1}^{L} \mathcal{O}_{x} t_{x}}\right\rangle_{s}=\frac{\sum_{x=1}^{L} P_{x}^{\mathrm{eq}}\left(s+\mathcal{O}_{x} u\right)^{\alpha-1}}{\sum_{x=1}^{L} P_{x}^{\mathrm{eq}}\left(s+\mathcal{O}_{x} u\right)^{\alpha}} .
$$

Using inversion technique found in [19], we transform Eq. (11), and find the PDF of the time average $\overline{\mathcal{O}}$

$$
f_{\alpha}(\overline{\mathcal{O}})=-\frac{1}{\pi} \lim _{\epsilon \rightarrow 0} \operatorname{Im} \frac{\sum_{x=1}^{L} P_{x}^{\mathrm{eq}}\left(\overline{\mathcal{O}}-\mathcal{O}_{x}+i \epsilon\right)^{\alpha-1}}{\sum_{x=1}^{L} P_{x}^{\mathrm{eq}}\left(\overline{\mathcal{O}}-\mathcal{O}_{x}+i \epsilon\right)^{\alpha}} .
$$

This is our main result so far, it is a very general formula for the distribution of time-averaged observables and is valid for a CTRW on a lattice. In the limit $\alpha \rightarrow 1$

$$
f_{\alpha=1}(\overline{\mathcal{O}})=\delta(\overline{\mathcal{O}}-\langle\mathcal{O}\rangle)
$$

which is the expected ergodic behavior. The opposite limit of $\alpha \rightarrow 0$ gives

$$
\lim _{\alpha \rightarrow 0} f_{\alpha}(\overline{\mathcal{O}})=\sum_{x=1}^{L} P_{x}^{\mathrm{eq}} \delta\left(\overline{\mathcal{O}}-\mathcal{O}_{x}\right) .
$$

This makes perfect physical sense, since when $\alpha \rightarrow 0$ the particle is localized for the whole duration of measurement in a single cell [20]. Note that our results can be easily generalized to dimensions higher than one.

In many applications the continuum behavior of the CTRW is important. Dynamically this limit corresponds to the behavior described by the fractional time FokkerPlanck equation [10, 11]. Taking the continuum limit of Eq. (12) we find

$$
f_{\alpha}(\overline{\mathcal{O}})=-\frac{1}{\pi} \lim _{\epsilon \rightarrow 0} \operatorname{Im} \frac{\int_{0}^{L} \mathrm{~d} x P^{\mathrm{eq}}(x)[\overline{\mathcal{O}}-\mathcal{O}(x)+i \epsilon]^{\alpha-1}}{\int_{0}^{L} \mathrm{~d} x P^{\mathrm{eq}}(x)[\overline{\mathcal{O}}-\mathcal{O}(x)+i \epsilon]^{\alpha}} .
$$

Here $P^{\text {eq }}(x) \mathrm{d} x$ is the equilibrium probability (in ensemble sense) of finding the particle in $(x, x+\mathrm{d} x)$ and $0<x<L$. When the random walk is coupled to a thermal heat bath with temperature $T$, in the presence of an external force field $F(x)$, the equilibrium of the ensemble is described by Boltzmann's statistics [9, 10]

$$
P^{\mathrm{eq}}(x)=\frac{\exp \left[-\frac{V(x)}{k_{b} T}\right]}{Z},
$$

where $Z$ is the partition function and $F(x)=$ $-\mathrm{d} V(x) / \mathrm{d} x$. As mentioned such an equilibrium is found

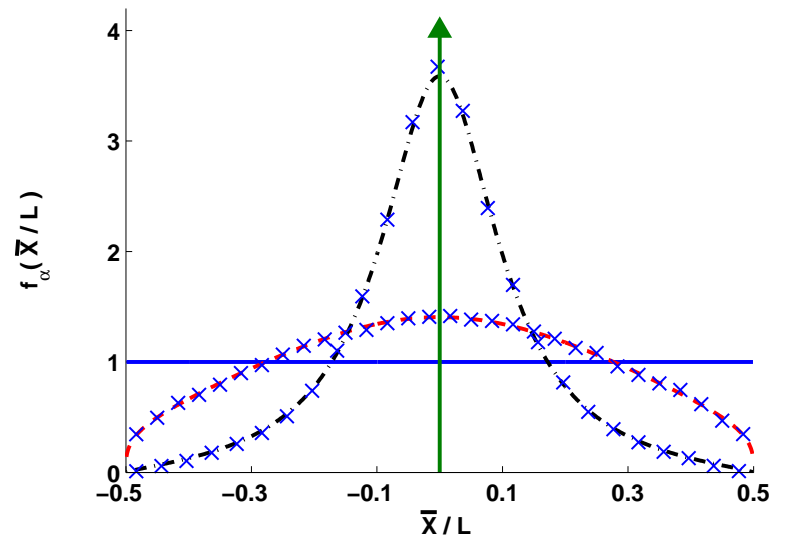

FIG. 1: The PDF of $\bar{X} / L$ for unbiased CTRW, simulations (crosses) versus theory (curves) Eq. (19). When $\alpha=1$ we find ergodic behavior and $\bar{X}=\langle X\rangle=0$ (i.e. the arrow symbolizing a delta function). For $\alpha=0.7$ (the dotted dashed curve) and $\alpha=0.3$ (the dashed curve) large fluctuations of time averages are observed. When $\alpha \rightarrow 0$ the PDF of $\bar{X}$ is uniform reflecting localization of the particle (solid line).

for the CTRW model when detailed balance conditions are imposed on $q_{x}$. Solving Eq. (15) we have

$$
f_{\alpha}(\overline{\mathcal{O}})=
$$

$\frac{\sin \pi \alpha}{\pi} \frac{I_{\alpha-1}^{<}(\overline{\mathcal{O}}) I_{\alpha}^{>}(\overline{\mathcal{O}})+I_{\alpha-1}^{>}(\overline{\mathcal{O}}) I_{\alpha}^{<}(\overline{\mathcal{O}})}{\left[I_{\alpha}^{>}(\overline{\mathcal{O}})\right]^{2}+\left[I_{\alpha}^{<}(\overline{\mathcal{O}})\right]^{2}+2 \cos \pi \alpha I_{\alpha}^{>}(\overline{\mathcal{O}}) I_{\alpha}^{<}(\overline{\mathcal{O}})}$

where

$$
I_{\alpha}^{<}(\overline{\mathcal{O}})=\int_{\overline{\mathcal{O}}<\mathcal{O}(x)} \mathrm{d} x P^{\mathrm{eq}}(x)|\overline{\mathcal{O}}-\mathcal{O}(x)|^{\alpha}
$$

and similarly for $I_{\alpha}^{>}(\overline{\mathcal{O}}), I_{\alpha-1}^{<}(\overline{\mathcal{O}})$ and $I_{\alpha-1}^{>}(\overline{\mathcal{O}})$. The integration domain in Eq. (18) is for $x$ satisfying the condition $\overline{\mathcal{O}}<\mathcal{O}(x)$.

As an example consider a particle in a domain $-L / 2<$ $x<L / 2$ undergoing an unbiased random walk. This is a free particle in the sense that no external field is acting on it. The time average of the particle's position $\bar{X}$ is considered, and obviously for this case $P^{\mathrm{eq}}(x)=1 / L$ for $-L / 2<x<L / 2$. Using Eq. (17) we find the PDF of the time-averaged position

$$
f_{\alpha}(\bar{X})=
$$




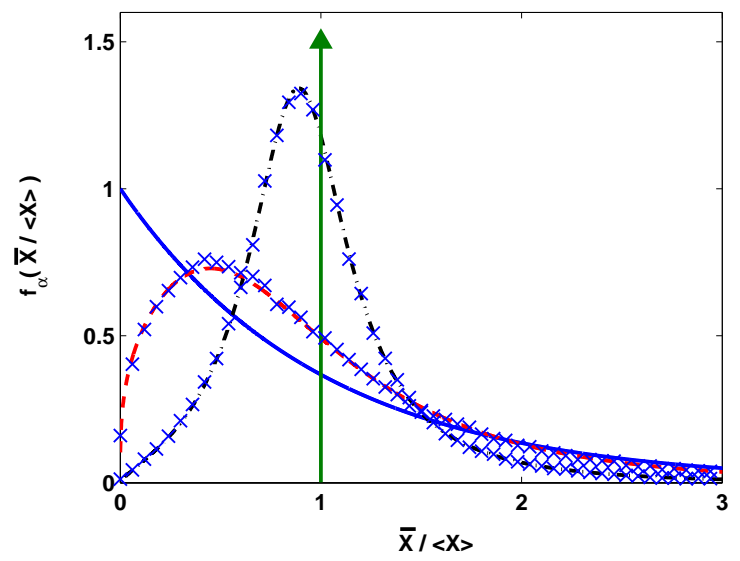

FIG. 2: Same as Fig. 1 for biased CTRW: now we show the PDF of $\bar{X} /\langle X\rangle$. The theoretical curves based on Eq. (20) perfectly agree with simulations (crosses) without fitting. A transition between ergodic behavior for $\alpha=1$ (the delta function) to localization behavior (solid curve $\alpha \rightarrow 0$ ) where the PDF of $\bar{X}$ decays exponentially is found.

$\frac{1}{L} \frac{N_{\alpha}\left(\frac{1}{4}-\frac{\bar{X}^{2}}{L^{2}}\right)^{\alpha}}{\left|\frac{1}{2}-\frac{\bar{X}}{L}\right|^{2(1+\alpha)}+\left|\frac{1}{2}+\frac{\bar{X}}{L}\right|^{2(1+\alpha)}+2\left|\frac{1}{4}-\left(\frac{\bar{X}}{L}\right)^{2}\right|^{1+\alpha} \cos \pi \alpha}$

where $N_{\alpha}=(1+\alpha) \sin \pi \alpha /(\pi \alpha)$. When $\alpha \rightarrow 1$ we have the ergodic behavior $\bar{X}=\langle X\rangle=0$ while $f_{\alpha \rightarrow 0}(\bar{X})=$ $1 / L$ for $|\bar{X}|<L / 2$ which is the uniform distribution, reflecting the mentioned localization of the particle in space when $\alpha \rightarrow 0$. In Fig. 1 comparison between our analytical results and numerical simulations [21] of the CTRW process with a fixed measurement time $t$, show excellent agreement without fitting.

As a second example consider a biased particle in the domain $0<x<\infty$ and in a constant force field $F>0$. Assuming the particle is in contact with a heat bath, with temperature $T$, Boltzmann's equilibrium is reached for an ensemble of particles, $P^{\mathrm{eq}}(x)=\exp \left(-F x / k_{b} T\right) / Z$. The PDF of $\bar{X}$ is found using Eq. (17)

$$
\begin{gathered}
f_{\alpha}(\bar{X})=\frac{\sin \pi \alpha}{\pi} \frac{F}{k_{b} T} \times \\
\frac{\Gamma(\alpha) e^{\tilde{x}} \tilde{x}^{\alpha}}{\left(\int_{0}^{\tilde{x}} \mathrm{~d} y e^{y}|y|^{\alpha}\right)^{2}+\Gamma^{2}(1+\alpha)+2 \Gamma(1+\alpha) \int_{0}^{\tilde{x}} \mathrm{~d} y e^{y}|y|^{\alpha} \cos \pi \alpha}
\end{gathered}
$$

where $\tilde{x}=F \bar{X} / k_{b} T$. When $\alpha \rightarrow 1$ we find ergodicity $f_{1}(\bar{X})=\delta(\bar{X}-\langle X\rangle)$ with $\langle X\rangle=k_{b} T / F$ while in the opposite limit $\alpha \rightarrow 0$, an exponential decay of the PDF of $\bar{X}$ is found: $\lim _{\alpha \rightarrow 0} f_{\alpha}(\bar{X})=\exp \left(-F \bar{X} / k_{b} T\right) / Z$ reflecting localization with a profile determined by the equilibrium density of many non-interacting particles. These behaviors are demonstrated in Fig. 2 .
We now discuss briefly the meaning of weak ergodicity breaking. In many situations in Physics a system is non-ergodic since its phase space is decomposed into regions of phase space where the system once starting in one region cannot explore the others. In this case time averages depend strongly on the initial condition of the system and there is no full exploration of phase space. In contrast for weak ergodicity breaking, the particle will visit each lattice cell many times, no matter what is its initial condition. Hence exploration of phase space is possible, and for this reason we were able to construct in this manuscript a general theory of non-ergodic statistical mechanics which is not sensitive to the initial conditions of the system. This has several implications, for example the joint PDF of occupation fractions Eq. (8) and the PDF of time averages Eqs. (15/17) are related to the population density $P^{\mathrm{eq}}(x)$. Therefore we may find a general relation between fluctuations of time averages and fluctuations of ensemble averages: using the small $u$ expansion of Eq. (11)

$$
\left\langle\overline{\mathcal{O}}^{2}\right\rangle-\langle\overline{\mathcal{O}}\rangle^{2}=
$$

$(1-\alpha)\left[\int_{0}^{L} \mathcal{O}(x)^{2} P^{\mathrm{eq}}(x) \mathrm{d} x-\left(\int_{0}^{L} \mathcal{O}(x) P^{\mathrm{eq}}(x) \mathrm{d} x\right)^{2}\right]$

while the average of $\overline{\mathcal{O}}$ is $\langle\overline{\mathcal{O}}\rangle=\langle\mathcal{O}\rangle=\int_{0}^{L} \mathcal{O} P^{\mathrm{eq}}(x) \mathrm{d} x$. For the example of a particle in a uniform force field $F$, when the physical observable is the position, we have $\langle\bar{X}\rangle=k_{b} T / F$ and $\left\langle\bar{X}^{2}\right\rangle-\langle\bar{X}\rangle^{2}=(1-\alpha)\left(k_{b} T / F\right)^{2}$.

To summarize we have obtained very general distributions of time averages of physical observables of weakly non-ergodic systems Eqs. (12/17). Unlike usual ergodic statistical mechanics where the time averages are equal to the ensemble averages, we find large fluctuations of time averages. Due to the large number of applications of the CTRW model, and the recent interest in weak ergodicity breaking in dynamics of single particles, our theory is likely to find its applications in many systems. Due to the deep relations between the stochastic CTRW model and other models of anomalous diffusion, e.g. the quenched trap model, and deterministic dynamics, our non-ergodic theory might find further profound justification.

Acknowledgment: This work was supported by the , Israel Science Foundation.

[1] J. C. Mauro, P. K. Gupta, and R. J. Loucks J. Chem. Phys. 126, 184511 (2007). De was named by M. Reiner after the prophetess Deborah, who in the Old Testament sings, ...the mountains flowed before the Lord... (Judges $5: 5)$.

[2] J. P. Bouchaud J. De Physique I 21705 (1992). 
[3] X. Brokmann, J. P. Hermier, G. Messin, P. Desbiolles, J. P. Bouchaud, and M. Dahan Phys. Rev. Lett., 90120601 (2003). G. Margolin, E. Barkai Phys. Rev. Letters 94 080601 (2005). G. Margolin, V. Protasenko, M. Kuno, E. Barkai Advances in Chemical Physics 133327 (2006).

[4] G. Bel, E. Barkai Phys. Rev. Lett. 94240602 (2005), ibid Phys. Rev. E 73016125 (2006).

[5] G. Bel, E. Barkai Europhysics Lett. 7415 (2006).

[6] E. Heinsalu, M. Patriarca, I. Goychuk, G. Schmidt and P. Hanggi Phys. Rev. E 73046133 (2006).

[7] M. A. Lomholt, I. M. Zaid, and R. Metzler Phys. Rev. Lett. 98, 200603 (2007).

[8] R.Klages, G.Radons, I.M.Sokolov (Eds.), Anomalous Transport: Foundations and Applications, Wiley-VCH, Weinheim (2007)

[9] J.P. Bouchaud and A. Georges, Physics Reports 195127 (1990).

[10] R. Metzler, J. Klafter, Phys. Rep. 3391 (2000).

[11] I. Sokolov, J. Klafter and A. Blumen Physics Today 55 48 (2002).

[12] P. Allegrini, M. Bologna, P. Grigolini, and B. J. West Phys. Rev. Lett. 99, 010603 (2007).

[13] J. Klafter, M.F. Shlesinger, G. Zumofen Physics Today 4933 (1996)
[14] H. Scher, G. Margolin, R. Metzler, and B. Berkowitz Geophys. Res. Lett. 29 10.1029/2001GL014123 (2002).

[15] S. B. Yuste and K. Lindenberg Phys. Rev. Lett. 87118301 (2001).

[16] H. Scher, M. F. Shlesinger, and J. T. Bendler, Physics Today 4426 (1991).

[17] For reflecting boundary conditions, it is easy to show that $P_{x}^{\mathrm{eq}}=N^{-1}\left[1 /\left(1-q_{x}\right)\right] \Pi_{k=2}^{x}\left(1-q_{k}\right) / q_{k}$ with the normalization $N=\sum_{x=1}^{L}\left[1 /\left(1-q_{x}\right)\right] \Pi_{k=2}^{x}\left(1-q_{k}\right) / q_{k}$.

[18] Related multi dimensional distributions were treated by Mathematicians: M. T. Barlow, J. Pitman, M. Yor Une extension multidimensionnelle de la loi de l'arc sinus. Séminaire de probabilités de Strasbourg 23294 (1989).

[19] C. Godreche, and J. M. Luck, J. of Statistical Physics 104489 (2001).

[20] We take the limit $t \rightarrow \infty$ before $\alpha \rightarrow 0$, otherwize the particles cannot reach equilibrium.

[21] In simulations we used: $\psi(\tau)=\alpha \tau^{-1-\alpha}$ when $\tau>1$ otherwise $\psi(\tau)=0, L=30,120000$ realizations, and the maximal measurement time (for $\alpha=0.3$ ) was $t=10^{16}$. For the biased CTRW shown in Fig. 2 we have $\langle X\rangle=16.7$ (lattice spacing is unity). 